\title{
The Physics of Soft Gamma Repeaters
}

\author{
C. Thompson \\ University of North Carolina, Department of Physics and Astronomy, \\ Chapel Hill, NC 27599, U.S.A.
}

\begin{abstract}
I describe the evidence that Soft Gamma Repeaters are magnetars-neutron stars in which a decaying magnetic field (rather than rotation) is the dominant source of free energy. The focus here is on the bursting emission of these sources and on direct physical diagnostics of very strong magnetic fields $\left(B \gtrsim 10 B_{Q E D}=4.4 \times 10^{14} \mathrm{G}\right)$. I also summarize the trapped fireball model of SGR outbursts, the influence of QED processes on their spectra and lightcurves, and the genetic connection between neutron star magnetism and the violent fluid motions in a collapsing supernova core.
\end{abstract}

\section{Introduction}

Neutron stars are known to be threaded by intense magnetic fields, through measurements of their polarized electromagnetic emissions (powered by rotation), pulsed X-ray emissions (powered by accretion), and changing spins (Manchester \& Taylor 1977; Lewin, van Paradijs, \& van den Heuvel 1995). These fields are almost certainly supported by electrical currents buried deep in the stellar interior. Although strong by terrestrial standards, the characteristic $10^{11}-10^{13} \mathrm{G}$ dipole magnetic fields of young neutron stars are, in a dynamical sense, quite weak. They contribute only $10^{-10}-10^{-8}$ of the hydrostatic pressure (when the effects of proton superconductivity in the stellar core are taken into account) and are probably too weak to be transported at a significant rate through the stellar interior (Baym et al. 1969; Pethick 1991; Goldreich \& Reisenegger 1992).

Substantial evidence has accumulated in recent years ${ }^{1}$ for neutron stars whose much stronger magnetic fields $\left(B_{\text {dipole }} \sim 10 B_{Q E D}=4.4 \times 10^{14} \mathrm{G}\right)$ decay significantly on a very short timescale $\left(\sim 10^{4} \mathrm{yr}\right)$. These magnetars were predicted to spin down much more rapidly than ordinary radio pulsars and should be elusive (although not necessarily impossible to detect) as pulsed radio sources. The defining property of a magnetar is that its decaying magnetic field outstrips its rotation as a source of energy for X-ray and particle emission-by some two orders of magnitude if the core field is as strong as $\sim 10^{2} B_{Q E D}$ (Thompson \& Duncan 1996, hereafter TD96). Magnetars have been associated (Duncan \&

\footnotetext{
${ }^{1}$ See Norris et al. (1991) for a review of the early literature on Soft Gamma Repeaters. We refer here to a representative sample of the more recent discoveries. A separate review (Thompson $2000 \mathrm{~b}$ ) focusses on the spin behavior and persistent emission of SGRs and Anomalous X-ray Pulsars in the magnetar model.
} 
Thompson 1992, hereafter DT92; Paczyński 1992; Thompson \& Duncan 1995, hereafter TD95) with a small peculiar class of neutron stars that emit extremely luminous bursts of hard X-rays and gamma-rays. These Soft Gamma Repeaters have in two cases (SGR 1806-20 and SGR 1900+14) been observed to emit more than $\sim 100$ short $(\sim 0.1 \mathrm{~s})$ bursts with a very broad range of fluences: $\sim 10^{5}$ for 1806-20 (Göğüs et al. 2000) and 10 ${ }^{4}$ for SGR 1900+14 (Göğüs et al. 1999).

SGRs are perhaps best known for two giant outbursts on March 5, 1979 and August 27, 1998 (from SGR 0526-66 and SGR 1900+14 respectively). These bursts, separated by almost 20 years, are nearly carbon copies of each other. They released $\sim 4 \times 10^{44}$ and $\sim 1 \times 10^{44} \mathrm{erg}$ in X-rays, respectively (Hurley et al. 1999a; Feroci et al. 1999; Mazets et al. 1999b and references therein), and had very similar and striking morphologies. Each was initiated by a very short and intense $(t \sim 0.1 \mathrm{~s})$ initial spike. The luminosity of this spike exceeded the classical Eddington luminosity - above which the outward force due to electron scattering exceeds the force of gravity: $L_{\text {edd }} \simeq 2 \times 10^{38} \mathrm{erg} \mathrm{s}^{-1}$ for a $1.4 M_{\odot}$ neutron star-by a factor $3 \times 10^{6}-10^{7}$ in the case of the March 5 event (Fenimore, Klebesadel, \& Laros 1996). The ensuing softer emission, which lasted 200-300 s and radiated somewhat more energy, had a much more stable temperature even though its luminosity exceeded $\sim 10^{4} L_{\text {edd }}$. In the March 5 event, this tail had a striking 8-second periodicity with a very large amplitude which was inferred to be the rotation period of the source (SGR 0526-66). The August 27 event exhibited a similar $5.16 \mathrm{~s}$ periodicity with an even larger amplitude.

The four known SGRs are also persistent X-ray sources of luminosity $10^{35}$ $10^{36} \mathrm{erg} \mathrm{s}^{-1}$ (Rothschild, Kulkarni, \& Lingenfelter 1994; Murakami et al. 1994; Hurley et al. 1999b; Woods et al. 1999). In two cases, persistent periodicities have been detected: $P=7.47$ s for SGR 1806-20 (Kouveliotou et al. 1998); and $P=5.16$ s for SGR $1900+14$ (Hurley et al. 1999c). This measurement of the spin preceded the August 27 event in the case of SGR 1900+14 and agreed with the periodicity detected in the giant outburst. Even more remarkably, both these SGRs have been observed to spin down at a very rapid rate with (coincidentally) nearly the same characteristic age of $P / \dot{P}=3000 \mathrm{yr}$ (Kouveliotou et al. 1998, 1999; Marsden, Rothschild, \& Lingenfelter 1999; Woods et al. 1999). The inferred polar strength of a time-variable dipole magnetic field exceeds $10^{15} \mathrm{G}$ in both cases. Nonetheless, the measured spindown luminosity $I \Omega \dot{\Omega}$ is smaller by two orders of magnitude than the persistent X-ray luminosity. In this situation, the inferred dipole field of both SGRs is reduced by a factor of $\sim 4$ to $4 \times 10^{14} \mathrm{G}$ if the star is a persistent source of Alfvén waves and particles with a luminosity comparable to $L_{X} \sim 10^{35} \mathrm{erg} \mathrm{s}^{-1}$ (Thompson \& Blaes 1998; Harding, Contopoulos, \& Kazanas 1999; Thompson et al. 1999). A final piece of evidence indicating that SGRs are young neutron stars comes from the association of all four-with varying degrees of certainty - with young supernova remnants (Kulkarni \& Frail 1993; Hurley et al. 1999b; Woods et al. 1999).

Anomalous X-ray Pulsars constitute a separate group of a half-dozen neutron stars that have been detected through their persistent X-ray pulsations but have never been observed to burst (Mereghetti \& Stella 1995; Duncan \& Thompson 1995; van Paradijs, Taam, \& van den Heuvel 1995; Mereghetti 2000). Nonetheless, AXPs share remarkably similar persistent X-ray luminosities $\left(L_{X} \sim 3 \times 10^{34}-10^{36} \mathrm{erg} \mathrm{s}^{-1}\right)$, spin periods $(P \sim 6-12 \mathrm{~s})$, and characteristic 
ages $\left(P / \dot{P} \sim 10^{3}-10^{5} \mathrm{yr}\right)$ with the SGRs. At least three are associated with young supernova remnants. This overlap between SGRs and AXPs in a threedimensional parameter space suggests that they are closely related. Combining both classes of sources, one roughly estimates the magnetar birth rate of SGRs/AXPs as $\sim 1 \times 10^{-3}$ per year (Thompson et al. 1999).

\section{Origins of Neutron Star Magnetism}

The idea of magnetars was motivated by the realization that the violent convective motions in a collapsing supernova core can strongly amplify the entrained magnetic field (Thompson \& Duncan 1993, hereafter TD93). The intense flux of neutrinos drives convection both in the central part of the core that is very thick to neutrino scattering and absorption (Pons et al. 1999, and references therein) and in a thin mass shell below the bounce shock where neutrino heating overcomes cooling (Janka \& Mueller 1996, and references therein). Balancing hydrodynamic and magnetic stresses, one deduces magnetic fields of $\sim 10^{15} \mathrm{G}$ and $\sim 10^{14} \mathrm{G}$, respectively (TD93; Thompson 2000a). The convection inside the neutrinosphere has an overturn time $\tau_{\text {con }}$ of a few milliseconds; the overturn time in the outer "gain" region is somewhat longer. The inner region will support a large-scale helical dynamo if the core is very rapidly rotating, with $P_{\text {rot }}<\tau_{\text {con }}$ (DT92), but not otherwise. It is also possible that rapid rotation by itself could amplify a magnetic field (Leblanc \& Wilson 1970) through the magnetic shearing instability (Balbus \& Hawley 1991) in the absence of convection if the outermost parts of the collapsing core became centrifugally supported.

A newborn neutron star experiences convection with a dimensionless ratio of convective kinetic energy to gravitational binding energy $\left(\varepsilon_{\text {con }} \sim 10^{-4}\right.$ ) that is some two orders of magnitude larger than in any previous phase driven by nuclear burning (TD93). (This is the relevant figure of merit because the gravitational binding energy and the magnetic energy are proportional under an expansion or contraction.) For this reason, neutron-star magnetic fields are probably not fossils from earlier stages of stellar evolution. The intense flux of neutrinos emanating from the neutron core induces rapid heating and $n-p$ transformations, thereby allowing magnetic fields stronger than $\sim 10^{14} \mathrm{G}$ to rise buoyantly through a thick layer of convectively stable material in less than the Kelvin time of $\sim 30 \mathrm{~s}$ (Thompson \& Murray 2000). As a result, the $10^{11}-10^{13} \mathrm{G}$ magnetic moments of ordinary radio pulsars, which do not appear to correlate with the axis of rotation, have a plausible origin (TD93) in a stochastic dynamo operating at slow rotation $\left(P_{\text {rot }} \gg \tau_{\text {con }}\right)$.

\section{Trigger and Energetics of SGR Bursts}

The bursts of SGR 1806-20 (Cheng et al. 1996; Göğüş et al. 2000) and SGR $1900+14$ (Göğüs et al. 1999) have some intriguing similarities with earthquakes and Solar flares. Their distribution of energies is a power-law with index $\mathrm{d} N / \mathrm{d} E$ $\propto E^{-1.6}$ that extends over some $4-5$ orders of magnitude in burst energy according to the most recent analyses; the distribution of waiting times is log-normal, peaking at $\sim 1$ day (Hurley et al. 1996; Gögüs et al. 1999; 2000). In the case of 1806-20, continuous monitoring by RXTE showed that the burst fluence accu- 
mulates in a piecewise-linear manner while the source is active (Palmer 1999). This indicates the existence of multiple active regions internal to the star, each of which induces activity in a neighboring region after dispensing its energy. Moreover, the star must be able to store a considerable amount of potential elastic or magnetic energy, amounting to $\sim 10^{45} \mathrm{erg}$ for the giant March 5 and August 27 flares. This should be compared with the maximum elastic energy $\sim 10^{44}\left(\theta_{\max } / 10^{-2}\right)^{2}$ erg that can be stored by the crust of a neutron star whose yield strain is $\theta_{\max }$. It should be emphasized at this point that a magnetic field stressing the outer Coulomb lattice of the star actually contains more available energy $\left(\delta B^{2} / 4 \pi\right)$ than is stored by the lattice itself $\left(\sim \frac{1}{2} \theta^{2} \mu\right.$, where $\mu$ is the shear modulus), by a factor $\sim 4 \pi \mu / B^{2}=10^{2}\left(B / 10 B_{Q E D}\right)^{-2}$. This allows a dipolar magnetic field of $\sim 10 B_{Q E D}=4.4 \times 10^{14} \mathrm{G}$ to power $\sim 30$ "March 5" outbursts during the lifetime of an individual SGR. Nonetheless, even with this amplification, it is not possible for a star composed of strange quark matter to retain enough potential magnetic energy to power the large March 5 and August 27 outbursts. The elastic energy of its crust is smaller than that of a neutron star by at least four orders of magnitude.

How precisely is energy injected into the magnetosphere of the neutron star? The very fast $(\sim \mathrm{ms})$ rise times, both of some short SGR bursts (Kouveliotou et al. 1987) and the giant outbursts (Fenimore et al. 1996; Hurley et al. 1999a; Mazets et al. 1999b), point to a localized and direct injection of energy. Indeed, much of the energy that is eventually radiated in the burst may have been injected on a much shorter timescale than the measured duration of the X-ray pulse. Direct evidence for this behavior comes from the intense initial spikes of the March 5 and August 27 events, which released a few tens of percent of the net outburst energy over $\sim 10^{-3}$ of the duration.

A magnetic field $B \gtrsim\left(4 \pi \theta_{\max } \mu\right)^{1 / 2} \sim 2 \times 10^{14}\left(\theta_{\max } / 10^{-3}\right)^{1 / 2} \mathrm{G}$ can fracture the crust but is far too weak to induce anything but a horizontal motion. As a result, energy is injected in the magnetosphere in two distinct regions. The motion will, in general, have a rotational component that creates tangential discontinuities in the magnetic field. A disturbance of the magnetosphere propagates at the speed of light, which is some 300 times the shear wave velocity $c_{\mu}$ in the deep crust. Thus, reconnection occurs rapidly and induces transverse Alfvén waves of frequency $\sim c / R_{N S}$ on the connecting closed loops of magnetic flux. These waves can dissipate effectively by cascading to high wavenumber through nonlinear interactions (Thompson \& Blaes 1998). The second dissipative region lies much farther out in the magnetosphere. The crustal motion (on a horizontal scale $\ell$ ) can be expected to excite shear waves of frequency $\nu \sim c_{\mu} / \ell$, which in turn couple to magnetospheric Alfvén modes at a radius $R_{\nu} \sim c / 3 \nu \sim 100 \ell$. This outer excitation may dominate if (for example) the fracture is buried deep in the crust, and has been identified with two bursts from SGR 1900+14 whose hard spectra resemble those of cosmological GRBs (Woods et al. 2000).

\subsection{Fracturing vs. Plastic Creep}

Finally, it should be noted that if the magnetic field in the deep crust exceeds $B_{\mu} \equiv(4 \pi \mu)^{1 / 2} \sim 6 \times 10^{15} \mathrm{G}$, lattice stresses are not able to compensate a departure from magnetostatic equilibrium and the crust must deform plastically (TD96). Indeed, the internal flux density above which the magnetic field is 
transported through the neutron star core on a timescale of $\sim 10^{4} \mathrm{yr}$ lies close to this value (TD96; Heyl \& Kulkarni 1998). This suggests that a magnetar is capable of two dissipative modes: one dominated by brittle fracturing and bright $\mathrm{X}$-ray outbursts, and a second dominated by plastic creep. These two modes correspond naturally to SGRs and to Anomalous X-ray Pulsars. In principle, both modes can operate simultaneously in the same star if its magnetic field is inhomogeneous.

\section{Diagnostics of Strong B}

\subsection{Hard Spikes of the March 5 and August 27 Events}

The initial spikes of the two giant outbursts had all the appearance of an expanding $e^{ \pm}$fireball carrying $\sim 10^{44} \mathrm{erg}$. (In the case of the March 5 event, $L \sim 3 \times 10^{6}-10^{7} L_{\text {edd }}$ and $T \sim 500 \mathrm{keV}$ : Mazets et al. 1999b; Fenimore et al. 1996). The peak luminosity is intermediate, on a logarithmic scale, between that of a thermonuclear X-ray flash and the bright $\gamma$-ray fireballs that are observed at cosmological distances. If the fireball contained comparable energy in radiation and in the rest energy of (baryonic) matter, then its duration could be expressed in terms of the radius $R\left(\tau_{e s}=1\right)$ of the scattering photosphere as $\Delta t \sim R\left(\tau_{e s}=1\right) / c \sim 1.4\left(E / 10^{44} \mathrm{erg}\right)^{1 / 2} \mathrm{~s}$, about 10 times the observed value. We conclude that the initial fireball must have, in fact, expanded relativistically and was powered by a very clean energy source.

The most obvious candidate is a magnetic field that experiences a sudden rearrangement. On energetic grounds, the (external) magnetic field must exceed $\sim 10 B_{Q E D}$ to power $\sim 10^{2}$ giant outbursts over $\sim 10^{4} \mathrm{yr}$. One might consider a hybrid model in which the energy is initially released inside the neutron star (in the form of crustal shear waves or torsional Alfvén waves in the liquid core) and only then transmitted to the magnetosphere. The large energy of the giant outbursts requires a large scale for this energy release and, hence, a low frequency for the excited mode. For example, a fracture of dimension $\ell \sim 1 \mathrm{~km}$ (a conservative lower bound) will excite a shear wave of frequency $\nu \sim 10^{3}(\ell / 1 \mathrm{~km})^{-1} \mathrm{~Hz}$. The resulting harmonic displacement $\xi$ of the crust will in turn excite oscillations of the dipolar magnetic field lines at a radius $R_{\nu} \sim c / 3 \nu \sim 10^{7}(\nu / \mathrm{kHz})^{-1} \mathrm{~cm}$. Because only a narrow bundle of the outer field is excited, the outward wave luminosity is a steep function of $\xi, \mathrm{d} E_{\text {wave }} / \mathrm{d} t \simeq \frac{1}{2} B_{\text {dipole }}^{2} R_{N S}^{2} c(2 \pi \xi \nu / c)^{8 / 3}$, or

$$
\frac{\mathrm{d} E_{\text {wave }}}{\mathrm{d} t} \simeq 2 \times 10^{44}\left(\frac{B_{\text {dipole }}}{10 B_{Q E D}}\right)^{2}\left(\frac{\xi}{0.1 \mathrm{~km}}\right)^{8 / 3}\left(\frac{\nu}{10^{3} \mathrm{~Hz}}\right)^{8 / 3} \mathrm{erg} \mathrm{s}^{-1},
$$

(Thompson \& Blaes 1998). For example, an elastic distortion of the crust of energy $\sim 10^{44} \mathrm{erg}$ corresponds to $\xi \sim 10^{-2} R_{N S} \sim 0.1 \mathrm{~km}$, and the luminosity approaches $10^{7} L_{\text {edd }}$ only if $B_{\text {dipole }} \sim 10^{15}\left(\nu / 10^{3} \mathrm{~Hz}\right)^{-4 / 3} \mathrm{G}$ !

Nonetheless, the short, $\sim 0.1 \mathrm{~s}$ duration of the intense initial spike of the March 5 and August 27 events provides direct evidence that internal (rather than external) magnetic stresses trigger these giant outbursts. A $10^{15} \mathrm{G}$ magnetic field will move the core material at a speed $\sim B / \sqrt{4 \pi \rho}$ through a distance $10 \mathrm{~km}$ in that period of time. By contrast, the fireball resulting from a sudden unwinding of the external field would last only $\sim R_{N S} / c \sim 10^{-4}$ s (TD95). 


\subsection{Soft Oscillatory Tails of the March 5 and August 27 Events}

After the initial hard spike, each of the two giant outbursts released an even greater amount of energy in an extended, oscillatory tail. The temperature during this phase was much more stable in spite of the hyper-Eddington flux, $L / L_{\text {edd }} \sim 10^{4}$ (e.g., see Mazets et al. 1999b). These observations suggest that a significant fraction of the initial burst of energy was trapped on closed magnetic field lines, which implies a strong lower bound to the surface dipole magnetic field, $B_{\text {dipole }}>2 \times 10^{14}\left(E_{\text {fireball }} / 10^{44} \mathrm{erg}\right)^{1 / 2}(\Delta R / 10 \mathrm{~km})^{-3 / 2}\left(1+\Delta R / R_{N S}\right)^{3} \mathrm{G}$ (TD95). A simple analytical model of a trapped fireball, cooling by the inward propagation of its cool boundary (TD95; Section 4.5), provides a remarkable fit to the extended August 27 lightcurve (Feroci et al., in preparation). Further evidence that the X-rays in the soft tail of the August 27 event were released close to the surface of the neutron star comes from the deep modulations at the $5.16 \mathrm{~s}$ rotational period of the neutron star. After $\sim 30 \mathrm{~s}$, each pulse exhibited 4 sharp subpeaks with a phase-coherent structure that appears to be the direct imprint of the multipolar structure of the star's magnetic field (Thompson et al. 1999).

Alternative models for storing the energy in the soft tail rapidly run into problems. This energy $\left(1-3 \times 10^{44} \mathrm{erg}\right)$ exceeds what can be plausibly retained in an elastic deformation of the crust. A strong core magnetic field $\left(B>10^{15} \mathrm{G}\right)$ could support a torsional Alfvén wave of this energy, but such a low-frequency wave would excite the magnetosphere at a very large radius $\left(\sim 10^{9} \mathrm{~cm}\right)-$ so large that it is difficult to see why the multipolar structure of the stellar field should be apparent in the lightcurve.

\subsection{Rotation and Persistent Emission}

Other key diagnostics of the magnetic field in the SGR sources are provided by measurements of spindown (Kouveliotou et al. 1998, 1999; Marsden et al. 1999; Woods et al. 1999) and persistent X-ray emission (TD96; Heyl \& Hernquist 1997; Thompson et al. 1999). The physical interpretation of the observed variability of the spindown (in both SGRs and AXPs) is covered more extensively in a separate review (Thompson 2000b). Three mechanisms can induce persistent Xray emission from a magnetar at a level of $\sim 10^{35} \mathrm{erg} \mathrm{s}^{-1}: 1$ ) heating of the core by ambipolar diffusion of the magnetic field, which increases the surface heat flux (TD96; Heyl \& Kulkarni 1998); 2) fracturing of the crust in the presence of small scale $(\sim 0.1 \mathrm{~km})$ magnetic irregularities, induced by the Hall electric field; and 3 ) twisting of the external magnetic field during giant outbursts, which drives a persistent thermionic current from the hot surface $(T \sim 0.5 \mathrm{keV})$ of a magnetar (Thompson et al. 1999). The heat conducted to the surface is enhanced by the reduction in magnetospheric opacities by the strong magnetic field. This effect was included by TD96, and its consequences have been examined by Heyl \& Hernquist (1999, and references therein) in much greater detail, especially as regards the increased transparency of a hydrogen envelope.

Two other direct diagnostics of the surface magnetic field of a magnetar are worth mentioning. 1) Afterglow radiation from the heated surface is exposed to a high-temperature fireball. The heated surface will release most of the absorbed thermal energy on the same timescale as it is absorbed (the observed duration of the outburst). The resulting luminosity increases monotonically with $B$ and is 
$\sim 10^{39} \times\left[\right.$ exposed area $\left./(10 \mathrm{~km})^{2}\right] \mathrm{erg} \mathrm{s}^{-1}$ for $T_{\text {fireball }} \sim 1 \mathrm{MeV}$ and $B \sim 10 B_{Q E D}$ (TD95). 2) There is absorption in the persistent emission at the ion-cyclotron resonance $\hbar \omega=2.8(Z / A)\left(B / 10 B_{Q E D}\right) \mathrm{keV}$. A direct measurement of the surface magnetic field would be provided by the simultaneous identification of a spin-flip transition: the two frequencies are very nearly degenerate for electrons but differ by a factor 2.8 for protons. This measurement is probably more feasible in AXPs, whose persistent emission has a much smaller nonthermal component than in SGRs.

\subsection{QED Processes: Their Radiative and Spectral Implications}

The transport of X-ray photons through a very strong (super-QED) magnetic field is determined by two coupled processes: Compton scattering and photon splitting $\gamma \rightarrow \gamma+\gamma$ (and merging $\gamma+\gamma \rightarrow \gamma$ ) (TD95). Even at very large scattering depth, the dielectric properties of the medium are dominated by vacuum polarization in the intense magnetic field. The normal modes of the electromagnetic field are then linearly polarized (e.g., see Mészáros 1992) with $\delta \mathbf{E} \perp \mathbf{B}_{0}$ in one case (the extraordinary or E-mode) and $\delta \mathbf{B} \perp \mathbf{B}_{0}$ in the other (the ordinary mode or O-mode).

The E-mode splits because, in this situation, its energy and momentum can be conserved by dividing it into two obliquely propagating $\mathrm{O}$-mode photons. ( $\mathrm{Or}$, at a lower rate, into a pair of E-mode and O-mode photons.) The O-mode is not able to split because its energy and momentum cannot be so conserved. ${ }^{2}$ In a vacuum, neither mode is able to split for the simple reason that the two daughter photons must remain colinear to conserve energy and momentum and there is no phase space for the process. In marked contrast with the strong $B^{6}$ scaling of the splitting rate in sub-QED magnetic fields, the splitting rate approaches a $B$-independent value in fields much stronger than $B_{Q E D}, \Gamma_{\mathrm{sp}}\left(\omega, B, \theta_{k B}\right)=$ $\left(\alpha_{\mathrm{em}}^{3} / 2160 \pi^{2}\right)\left(m_{e} c^{2} / \hbar\right)\left(\hbar \omega / m_{e} c^{2}\right)^{5} \sin ^{6} \theta_{k B}$ (Adler 1971; Thompson \& Duncan 1992). Here, $\alpha_{\mathrm{em}} \simeq 1 / 137$, and $\theta_{k B}$ is the angle between the photon's wavevector and the background magnetic field. This implies immediately that an E-mode photon propagating a distance $R_{N S} \sim 10 \mathrm{~km}$ through a super-QED B-field will split if $\hbar \omega>38\left(R_{N S} / 10 \mathrm{~km}\right)^{-1 / 5} \mathrm{keV}$ (TD95; Baring 1995).

It is well known that Compton scattering becomes strongly anisotropic in a background magnetic field, with a strongly frequency-dependent cross-section (Mészáros 1992). Unlike in a plasma, both vacuum modes interact resonantly with an electron (or positron) at the Landau frequencies. Near the surface of the star, the energy of the first Landau excitation $\left[\simeq\left(2 B / B_{Q E D}\right)^{1 / 2} m_{e} c^{2}\right.$ when $\left.B \gg B_{Q E D}\right]$ is much higher than the temperature of the emerging X-radiation. In this situation, there is a strong suppression of the E-mode's scattering crosssection: $\sigma_{E}=\left(\omega m_{e} c / e B_{0}\right)^{2} \sigma_{T}$ (e.g., see Herold 1979). This suppression greatly

\footnotetext{
${ }^{2}$ These selection rules depend essentially on the inequality $n_{O}>n_{E}$ between the indices of refraction of the two modes. Note that both $n_{O}$ and $n_{E}$ depart only very slightly from unity even in magnetic fields as strong as $\sim 10^{16} \mathrm{G}$. The inequality is reversed, $n_{E}>n_{O}$, when plasma dominates the dielectric properties of the medium, but, in such a situation, the particle density is enormous and the photons will, in practice, follow a Planckian distribution. The problem of calculating the emergent spectra of SGR bursts focusses on much lower temperatures and densities where departures from local thermodynamic equilibrium can occur.
} 
increases the radiative transport rate close to the neutron star-both from its surface (Paczyński 1992; Ulmer 1994) and across the confining magnetic field lines of a trapped fireball (TD95).

However, even in the region where the E-mode is able to stream freely, the O-mode can still undergo many Compton scatterings and relax to a BoseEinstein distribution. This permits a very simple generalization of the LTE diffusion formalism to an anisotropic, magnetized plasma at large E-mode scattering depth in which the photon energy and number fluxes are expressed as linear superpositions of gradients in the temperature $T$ and photon chemical potential $\mu$ (TD95). From our discussion of photon splitting, there is clearly a critical temperature above which the distributions of the E- and O-modes both become thermal, which works out to $T_{\mathrm{sp}}=11\left(R_{N S} / 10 \mathrm{~km}\right)^{-1 / 5} \mathrm{keV}$ (TD95).

Scattering at the electron-cyclotron resonance can probably be neglected during outbursts from a magnetar: the resonance sits at a large radius $8 R_{N S}$ $\left(B_{\text {dipole }} / 10 B_{Q E D}\right)^{1 / 3}(\hbar \omega / 10 \mathrm{keV})^{-1 / 3}$ where the outflowing photons are sufficiently collimated to suppress $\tau_{c y c}$ below unity. Scattering at the ion-cyclotron resonance has a significant optical depth if electrons and ions dominate the electron-scattering opacity: it is $\sim\left(\pi / 4 \alpha_{e m}\right) n_{e} \sigma_{T} R\left(B / B_{Q E D}\right)^{-1}$ in a dipolar magnetic field. The main effect will be to convert photons between the E- and $\mathrm{O}$-modes and to increase significantly the opacity of the E-mode at low frequencies. (This may be relevant to the $<7 \mathrm{keV}$ suppression of the radiative flux found by Ulmer et al. 1995 in the bursts of SGR 1806-20.)

\subsection{Cooling of a Trapped Fireball}

The bubble of $e^{ \pm}$pairs and $\gamma$-rays that powers a short SGR burst (of duration $\sim 0.1 \mathrm{~s}$ ) is inferred to have a temperature $\sim 0.1 \mathrm{keV}$ (for a confining volume $\sim(10 \mathrm{~km})^{3}$ and energy $\left.\sim 10^{41} \mathrm{erg}\right)$. The corresponding temperature for a giant outburst is closer to $\sim 1 \mathrm{MeV}$. The optical depth to scattering across such a bubble is huge, approaching $\sim 10^{10}$ in the latter case. It is clear that the bubble cannot cool by simple radiative diffusion from its center: that would take $\sim 10^{3}-10^{4}$ times the observed burst duration. The bubble cools instead as a sharp temperature gradient develops just inside its outer boundary, and this boundary propagates inward as a cooling wave (TD95). If the magnetic field is predominantly dipolar, then the radiative flux across the field is concentrated near the surface of the star: the opacity of the E-mode scales as $B^{-2} \propto R^{6}$. A cylindrical bundle of field lines containing relativistic plasma therefore has a radiative area (and luminosity) that decreases linearly with time, as is observed in a number of short SGR bursts (e.g., Mazets et al. 1999a). If higher multipoles dominate the near magnetic field, then the opacity will be much more uniform over the surface of the fireball. Parametrizing the radiative area in terms of the remaining fireball energy as $A \propto E_{\text {fireball }}^{\alpha}$, the luminosity works out to

$$
L_{X}(t)=L_{X}(0)\left(1-\frac{t}{t_{\text {evap }}}\right)^{\alpha /(1-\alpha)} .
$$

Here, $t_{\text {evap }}$ is the time at which the cooling wave propagates to the center of the fireball and the fireball evaporates. This analytic law provides an excellent fit to the extended lightcurve of the August 27 event for $\alpha \sim 0.7$ (Feroci et al., in preparation). 
Further evidence for the presence of a trapped fireball comes from the sharply peaked subpulses within the oscillatory tail of the August 27 event. This pattern requires a collimated, quasi-hydrodynamical outflow of the X-radiation, which was suggested to be the consequence of Compton scattering in intense magnetic fields (TD95; Thompson et al. 1999). The rapid rise of the E-mode opacity with radius provides a mechanism for self-collimation: if baryonic matter is suspended in the magnetosphere by the hyper-Eddington radiative flux, then E-radiation will escape by pushing this matter to the side. In addition, a significant fraction of the E-mode flux near the E-mode photosphere is converted to the O-mode by scattering and by photon splitting (Miller 1995; TD95). The O-mode flows hydrodynamically along the magnetic field even in the presence of a tiny amount of matter, $\dot{M} c^{2} / L_{O} \sim\left(G M_{N S} / R_{N S} c^{2}\right)^{-1}\left(L_{O} / L_{e d d}\right)^{-1}$. Further collimation occurs if the photosphere is aligned with extended (dipolar) magnetic field lines.

\section{References}

Adler, S. L. 1971, Ann. Phys., 67, 599

Balbus, S. A., \& Hawley, J. F. 1991, ApJ, 376, 214

Baring, M. A. 1995, ApJ, 440, L69

Baym, G., Pethick, C., \& Pines, D. 1969, Nature, 224, 674

Cheng, B., Epstein, R. I., Guyer, R. A., \& Young, A. C. 1996, Nature, 382, 518

Duncan, R. C., \& Thompson, C. 1992, ApJ, 392, L9 (DT92)

Duncan, R. C., \& Thompson, C. 1995, in High Velocity Neutron Stars and Gamma-Ray Bursts, eds. R. E. Rothschild \& R. E. Lingenfelter (New York: AIP), 111

Fenimore, E. E., Klebesadel, R. W., \& Laros, J. G. 1996, ApJ, 460, 964

Feroci, M., et al. 1999 ApJ, 515, 9

Feroci, M., et al., in preparation

Goldreich, P., \& Reisenegger, A. 1992, ApJ, 395, 250

Göğüş, E., et al. 1999, ApJ, 526, L93

Göğüs, E., et al. 2000, ApJ, submitted

Harding, A. K., Contopoulos, I., \& Kazanas, D. 1999, ApJ, 525, L125

Herold, H. 1979, Phys. Rev. D, 19, 2868

Heyl, J. S., \& Hernquist, L. 1997, ApJ, 489, L67

Heyl, J. S., \& Hernquist, L. 1999, MNRAS, 300, 599

Heyl, J. S., \& Kulkarni, S. R. 1998, ApJ, 506, L61

Hurley, K. J., McBreen, B., Rabbette, M., \& Steel, S. 1994, A\&A, 288, L49

Hurley, K., et al. 1999a, Nature, 397, 41

Hurley, K., et al. 1999b, ApJ, 510, L107

Hurley, K., et al. 1999c, ApJ, 510, L111

Janka, H.-T., \& Mueller, E. 1996, A\&A, 306, 167

Kouveliotou, C., et al. 1987, ApJ, 322, L21 
Kouveliotou, C., et al. 1998, Nature, 393, 235

Kouveliotou, C., et al. 1999, ApJ, 510, L115

Kulkarni, S. R., \& Frail, D. A. 1993, Nature, 365, 33

Leblanc, J. M., \& Wilson, J. R. 1970, ApJ, 161, 541

Lewin, W. H. G., van Paradijs, J., \& van den Heuvel, E. P. J. 1995, X-ray Binaries (Cambridge: University Press)

Manchester, R. N., \& Taylor, J. H. 1977, Pulsars (San Francisco: Freeman)

Marsden, D., Rothschild, R. E., \& Lingenfelter, R. E. 1999, ApJ, 520, L107

Mazets, E. P., et al. 1999a, Astron. Lett., 25, 628

Mazets, E. P., et al. 1999b, Astron. Lett., 25, 635

Mereghetti, S. 2000, in The Neutron Star - Black Hole Connection, eds. C.

Kouveliotou, J. van Paradijs, \& J. Ventura (Dordrecht: Kluwer), in press

Mereghetti, S., \& Stella, L. 1995, ApJ, 442, L17

Mészáros, P. 1992, High-Energy Radiation from Magnetized Neutron Stars (Chicago: University Press)

Miller, M. C. 1995, ApJ, 448, L29

Murakami, T., et al. 1994, Nature, 368, 127

Norris, J. P., Hertz, P., Wood, K. S., \& Kouveliotou, C. 1991, ApJ, 366, 240

Paczyński, B. 1992, Acta Astron., 42, 145

Palmer, D. M. 1999, ApJ, 512, L113

Pethick, C. J. 1992, in Structure and Evolution of Neutron Stars, eds. D. Pines, R. Tanagaki, \& S. Tsuruta (Redwood City: Addison-Wesley), 115

Pons, J. A., et al. 1999, ApJ, 513, 780

Rothschild, R. E., Kulkarni, S. R., \& Lingenfelter, R. E. 1994, Nature, 368, 432

Thompson, C. 2000a, ApJ, in press

Thompson, C. 2000b, in Pulsar Astronomy - 2000 and Beyond, eds. M. Kramer,

N. Wex, \& R. Wielebinski (San Francisco: AIP), in press

Thompson, C., \& Blaes, O. 1998, Phys. Rev. D, 57, 3219

Thompson, C., \& Duncan, R. C. 1992, in Compton Gamma Ray Observatory, eds. M. Friedlander, N. Gehrels, \& R. J. Macomb (New York: AIP), 1085

Thompson, C., \& Duncan, R. C. 1993, ApJ, 408, 194 (TD93)

Thompson, C., \& Duncan, R. C. 1995, MNRAS, 275, 255 (TD95)

Thompson, C., \& Duncan, R. C. 1996, ApJ, 473, 322 (TD96)

Thompson, C., et al. 1999, astro-ph/9908086, preprint

Thompson, C., \& Murray, N. W. 2000, ApJ, submitted

Ulmer, A. 1994, ApJ, 437, L111

Ulmer, A., et al. 1993, ApJ, 418, 395

van Paradijs, J., Taam, R. E., \& van den Heuvel, E. P. J. 1995, A\&A, 299, L41

Woods, P. M., et al. 1999, ApJ, 519, L139

Woods, P. M., et al. 2000, ApJ, in press 